\title{
Philosophiques
}

\section{Entre la rime et la raison. Précis de L'Esprit et la nature}

\section{Daniel Laurier}

Volume 30, numéro 2, automne 2003

URI : https://id.erudit.org/iderudit/008648ar

DOI : https://doi.org/10.7202/008648ar

Aller au sommaire du numéro

Éditeur(s)

Société de philosophie du Québec

ISSN

0316-2923 (imprimé)

1492-1391 (numérique)

Découvrir la revue

Citer cet article

Laurier, D. (2003). Entre la rime et la raison. Précis de L'Esprit et la nature.

Philosophiques, 30(2), 407-410. https://doi.org/10.7202/008648ar d'utilisation que vous pouvez consulter en ligne.

https://apropos.erudit.org/fr/usagers/politique-dutilisation/ 


\title{
Entre la rime et la raison. Précis de L'Esprit et la nature ${ }^{1}$
}

\author{
DANIEL LAURIER \\ Université de Montréal \\ daniel.laurier@umontreal.ca
}

Le petit livre évoqué dans le titre a été écrit entre 1993 et 2001. Il s'agit en fait d'un recueil d'articles qui sont unifiés par un thème: celui de la nature des contenus intentionnels. Je n'y défends pas de théorie particulière de l'intentionnalité. La teneur de ces articles est tantôt critique, tantôt spéculative et exploratoire. Dans ma présentation, je ne vais pas essayer d'en résumer le contenu. Je vais plutôt essayer d'expliquer ce que je pense, rétrospectivement, pouvoir en retenir.

Un présupposé qui traverse tout le livre est que le contenu intentionnel est essentiellement normatif. J'entends par là que je n'envisage pas qu'il soit sérieusement possible d'expliquer le contenu intentionnel sans faire référence, d'une manière ou d'une autre, à la notion normative de rationalité. Manifestement, c'est un présupposé que j'ai hérité de Davidson et qui aurait certainement besoin d'être clarifié et défendu. Mais je ne fais pratiquement aucune tentative, dans ce livre, pour le clarifier ou le défendre. Je me contente de le tenir pour acquis.

Le livre peut être divisé, grosso modo, en deux parties. Dans la première, je traite essentiellement de la notion de fonction téléologique et de la manière dont Fred Dretske a proposé de l'exploiter pour rendre compte de la nature des contenus intentionnels et de leur efficacité causale. Dans la deuxième, j'essaie d'en dire un peu plus sur les formes et les limites du naturalisme philosophique et sur la manière dont on pourrait espérer progresser dans la compréhension du phénomène de l'intentionnalité.

Je vais faire quelques remarques sur chacun de ces deux aspects, en essayant de rester le plus près possible de la ligne directrice générale, passant sous silence un certain nombre de points qui me semblent aujourd'hui contestables, parfois même obscurs.

L'examen de la théorie de l'intentionnalité de Dretske est précédé d'une discussion concernant l'analyse du concept de fonction. Une condition pour que la notion de fonction puisse avoir la vedette dans une explication du contenu intentionnel est qu'on lui reconnaisse un aspect normatif. La question est donc de savoir s'il est possible d'avoir une explication naturaliste de la notion de fonction qui soit effectivement capable de

1. Laurier, Daniel (2002), L'Esprit et la nature, Montréal, Presses de l'Université de Montréal. 
lui conférer une force normative. Une condition nécessaire pour cela, c'est qu'il soit possible qu'une chose ait une fonction même si elle ne la remplit pas et même si elle est incapable de la remplir. Je montre qu'une explication sélectionniste de la notion de fonction dans l'esprit d'auteurs tels que Neander et Millikan peut satisfaire à cette condition tout en respectant un certain nombre d'autres contraintes. Mais on ne peut prétendre qu'une telle explication est suffisante pour rendre compte de la dimension normative du concept de fonction sans enfreindre le principe de Hume selon lequel on ne peut inférer ce qui doit être de ce qui est.

Je dois dire qu'aujourd'hui, cette objection me semble beaucoup moins convaincante qu'il y a dix ans. Le principe de Hume me semble beaucoup moins clair qu'auparavant, et je pense qu'il y a peut-être des manières de le contourner, mais que cela exigerait un examen très minutieux non seulement de la distinction entre faits et normes, mais aussi de la notion d'inférence elle-même. Le même problème refait surface plus loin, dans la deuxième partie du livre, et le fait qu'il ne soit pas réellement traité (en tout cas pas de manière sérieuse) jette selon moi un doute sur la validité de mes conclusions concernant le destin du naturalisme.

Après cette discussion sur le concept de fonction, vient une assez longue étude critique de la théorie du contenu intentionnel proposée par Dretske en 1988, dans Explaining Behavior. C'est certainement le chapitre le plus difficile à lire et le plus technique qu'on trouve dans ce livre. C'est aussi le plus long. L'idée de base de Dretske est qu'un état interne d'un organisme est considéré comme une représentation du fait que $p$ lorsqu'il a pour fonction d'indiquer que $p$, c'est-à-dire, grosso modo, lorsqu'il a pour fonction de nécessiter nomiquement que $p$. Son problème, c'est de développer une explication plausible de la manière dont un état interne d'un organisme peut acquérir pareille fonction et de montrer que le fait qu'un état interne ait acquis une telle fonction peut contribuer à expliquer causalement certains comportements de l'organisme en question. L'effet cumulatif de mes commentaires sur Dretske est assez destructeur. Il n'y a à peu près aucun aspect de sa théorie qui puisse être sauvé, à moins de la réinterpréter comme une théorie qui vise à rendre compte non pas du contenu des croyances et des désirs, mais de celui d'états intentionnels beaucoup plus primitifs (et, il faut bien le dire, beaucoup moins bien définis). Mais même en admettant qu'il y a effectivement une catégorie de contenus intentionnels qui vérifient son analyse de la notion de contenu, sa théorie ne permettrait pas de leur conférer de pertinence causale ou explicative.

Je ne considère pas avoir montré qu'il est impossible de rendre compte du contenu intentionnel en s'appuyant sur la notion de fonction téléologique. Il y a d'autres formes de téléosémantique que je n'ai pas examinées dans le détail. Pour autant que je puisse en juger, celle de Millikan, en particulier, échappe à bon nombre des critiques que j'ai soule- 
vées contre Dretske. Je regrette un peu, rétrospectivement, de ne pas lui avoir accordé toute l'attention qu'elle mérite.

Je pense que ce qui m'a empêché de le faire, ou en tout cas d'avoir la motivation pour entreprendre de le faire, c'est ma conviction qu'il y a des objections de principe qui s'appliquent de manière générale à tout projet de naturalisation de l'intentionnalité. J'ai essayé, dans la deuxième partie du livre, de faire écho à ces inquiétudes plus générales en tentant, dans un premier temps, de caractériser le naturalisme philosophique de manière un peu plus complète.

Cela m'a amené à suggérer que le trait essentiel du naturalisme est de nier l'existence de normes non naturelles. Cela permet de distinguer deux formes de naturalisme concernant l'intentionnalité, soit une forme radicale, qui soutient que les phénomènes intentionnels ne sont absolument pas normatifs, et une forme modérée, qui admet qu'ils le sont, mais soutient que les normes requises pour en rendre compte sont «naturelles». Dretske et Millikan tombent naturellement dans la famille des naturalistes intentionnels modérés. Je ne vois que Quine (et peut-être certaines phases de Fodor) qui puisse appartenir à la catégorie des naturalistes intentionnels radicaux.

Je présente ensuite une distinction entre deux types de contenus intentionnels: des contenus conceptuels et des contenus non conceptuels. Cette distinction me permet non seulement de satisfaire à l'intuition qui veut que les enfants pré-linguistiques et les animaux soient capables d'être dans des états intentionnels, mais aussi de faire ressortir le fait que ceux qui, comme Davidson, s'opposent à la possibilité de naturaliser les contenus intentionnels le font généralement en tenant pour acquis que les contenus intentionnels ne peuvent être que conceptuels. L'objection fondamentale est qu'on ne saurait naturaliser les contenus conceptuels, parce que, pour en rendre compte, il faut invoquer la notion normative de rationalité et, qu'inversement, on ne peut rendre compte des normes de la rationalité sans invoquer la notion de contenu conceptuel.

À première vue, cette objection dépend d'une conception «constructiviste» de la rationalité, selon laquelle il n'y aurait pas de normes de rationalité si nous ne pouvions pas les concevoir. Mais il reste que même si on rejette l'idée que la rationalité dépend de l'intentionnalité conceptuelle, c'est le naturaliste qui a le fardeau de produire une explication de la rationalité conforme à ses exigences; or aucune explication de ce genre ne semble vouloir poindre à l'horizon.

Cela me conduit à la conclusion que le naturalisme intentionnel radical est la seule forme viable de naturalisme intentionnel. C'est une forme de naturalisme qui a des conséquences à mon avis contre-intuitives, mais qui ne remet pas nécessairement en question les conceptions fondamentales que nous avons de nous-mêmes. Car on peut être naturaliste intentionnel radical sans être naturaliste "tout court ", c'est-à-dire sans soutenir qu'il n'y a pas de normes non naturelles du tout. Le naturaliste intentionnel 
radical soutien simplement qu'il n'y a pas de normes qui soient constitutives des phénomènes intentionnels.

Dans le dernier chapitre, je suggère qu'on pourrait faire quelques progrès dans la compréhension de l'intentionnalité si on essayait de diviser les problèmes. Je propose, en particulier, qu'on pourrait envisager d'expliquer simultanément les contenus conceptuels et les normes de rationalité en s'appuyant sur la notion de contenu non conceptuel, et j'indique comment la théorie de Brandom pourrait être interprétée comme un pas dans cette direction.

Une conséquence de cette proposition, c'est que si on ne peut plus soutenir qu'il y a entre l'intentionnalité non conceptuelle et la rationalité le même genre d'interdépendance qu'entre l'intentionnalité conceptuelle et la rationalité, alors il y a peut-être une chance de produire une explication naturaliste des contenus non conceptuels. Ce qu'on peut dire, en tout cas, c'est que s'il y a une objection de principe à la naturalisation des contenus non conceptuels, elle ne pourra pas être du même type que celle soulevée par Davidson contre la naturalisation des contenus conceptuels.

Je pense qu'il y a une telle objection, objection qui s'applique en fait à toute forme de naturalisme modéré (qu'il concerne ou non les phénomènes intentionnels). Mais cette objection n'est pas formulée dans L'Esprit et la nature (on la retrouve dans un article ultérieur ${ }^{2}$ ). Elle consiste tout simplement à montrer que cette forme de naturalisme ne peut qu'être triviale.

Donc, pour moi, l'intérêt d'invoquer les contenus non conceptuels n'est pas que cela pourrait contribuer à naturaliser l'intentionnalité, mais tout simplement que cela me semble une manière de progresser dans la compréhension des structures de l'intentionnalité.

Depuis que mon livre a été publié, je me suis rendu compte qu'il y a encore beaucoup de travail conceptuel à faire avant qu'on ne soit en mesure de déterminer s'il y a réellement des fruits dans ce verger.

2. "Quatorze observations topographiques sur les contenus et les normes", Facta philosophica, 2002, no 4, p. 177-199. 